\title{
Freedom vs. Constraint
}

By N.N.

- Desideratum

\section{Evernote keywords: issues tagged for first-priority treatment in this entry}

- altruism $\lambda$ egotism

- anonymity, masking, veiling

- arranged marriages

- $\operatorname{art}(\mathrm{s})$, artistic freedom, artist as enemy

- Article 230

- auto-documentary

- censorship, self-sensorship

- challenging the givens/stereotype, provocation

- chewing gum

- clubs

- collective $\lambda$ Individuality vs. Collectivity

- commitment / iltizām

- common destiny

- conscience

- duty, sense of duty/responsibility, Freedom vs. Duty/Responsibility

- ego, selfishness, Egotism vs. Altruism

- family: constraints imposed by family, familiy honour, family pressure, family values, familial solidarity

- forced social gatherings

- formation of the Self, individual trajectories, individualisation, individuation

- freedom $(Q T h)$, lack of freedom, lack of freedom of expression, lack of dignity

- Freedom vs. Duty/Responsibility $\lambda$ duty

- guards, guardians

- hackers, hacking

- helplessness

- homosexuality: see $\nearrow$ prosecution
- impotence, feeling of powerlessness

- individual appropriation

- individual happiness

- individual $v s$. state

- individual trajectories, individualisation, individuation $\lambda$ formation of the Self

- limits

- "loose morals"

- narrow spaces, closed rooms

- "offending public morals"

- personal $=$ national dignity

- Personal vs. Collective

- potentials

- premarital sex

- pressure

- printers as censors

- private, privacy

- project(s)

- prosecution of homosexuality

- prosecutor's speech in front of CairoAppeal Court

- public

- Public $=$ Private

- public condemnation

- public image

- public insults

- public morals / morality, public shaming

- public space, Public vs. Private, Public space vs. Private space

- public unrest

- puritanism

- qillit adab

- quest for one's self

- questions

- reaction

- reaction vs. lack of reaction

- reactions to al-Sīsī's speech 13 August

- "red lines" 
- relatives

- relativism

- religious police

- respect

- respectable citizens

- responsibility $\lambda$ duty

- responsible citizens $v s$. distrustful authorities

$-\operatorname{ritual}(\mathrm{s})$

- rules

- self

- self-assurance (via apologetics), selfconfidence

- self-awareness, $\sim$ and reflection

- sense of duty / responsibility $\nearrow$ duty

- sexual frustration

- shame

- singles

- social and culture choices

- social mores, norms, rules, trends, values

- social control, obligations, social pressure

- stagnation

- stereotypes $\lambda$ challenging

\section{Entries pointing here}

ARRAYS - Apartment Wanted $\downarrow$ Commemoration $/$ Memorial Days $\downarrow$ Conversions $\downarrow$ Court Trials Crowdfunding Dual Identities / Masking $\downarrow$ Father Figures Garbage Hashish $\downarrow$ Mobile Phones $\diamond$ Pop Music $\diamond$ Prison $\diamond$ Psychiatrists $\diamond$ Self-help $\downarrow$ Tourist Resorts $\downarrow$ Uber

COdes - Beautiful vs. Ugly Male vs. Female True vs. False Young vs. Settled

CODES COLLAPSED - True $=$ False $($ Life in Limbo $)$

- strong will

- submission

- subversion, subversive, subversive energy of music

- suffering and discomfort

- suffocation

- suicide, suicide rates

- taboos

- $t a^{\prime} d \bar{l} b$

- tamkin > empowerment

- timidity

- "torn between two women"

- tradition, tradition's pressure, Tradition vs. Freedom

- transfiguration

- translocate

- trap

- true identity/self

- uncertainty

- undecided

- unmarried women living alone

- values

- vulnerability

- What to do?

- women's liberation 